\title{
A microcomputer-controlled solid-state dark adaptometer
}

\author{
PETER A. O'MARA, HARRY ZWICK, and CHARLES W. VAN SICE \\ Division of Ocular Hazards, Letterman Army Institute of Research \\ Presidio of San Francisco, California 94129
}

\begin{abstract}
A low-cost microprocessor-controlled light-emitting diode (LED) dark adaptometer is described. The apparent intensities of red and green stimuli are controlled by changing the duty cycles of LED display elements which are operated at constant pulse repetition rates. The psychophysical method of limits is used to obtain threshold data. Stimulus parameters and test procedures are under software control. The design utilizes programmable integrated circuitry that may be used with a variety of microprocessors.
\end{abstract}

Instruments used to measure the course of dark adaptation typically have an adjustable optical density wedge or a series of filters to change the brightness of a constant-intensity filament light source. These devices are often mechanically complicated and expensive when automated. An inexpensive microprocessor-controlled dark adaptometer which uses red and green light-emitting diodes (LEDs) as test stimuli has been developed at the Letterman Army Institute of Research (Zwick, Biggs, O'Mara, and Van Sice, 1980). This device varies the apparent luminance of the test stimuli by changing the duty cycles of LEDs that are operated at a fixed frequency $(100 \mathrm{~Hz})$ above the critical flicker fusion threshold. This instrument permits simple software modification of test parameters and provides on-line data reduction with graphic output of the adaptation functions. This paper describes the hardware, software, and method of operation of the LED dark adaptometer.

\section{HARDWARE}

The LED adaptometer utilizes readily available, lowcost, integrated circuits (ICs) to interface the bus of an $8080 \mathrm{~A}$ (or other) microprocessor to an LED target display (Figure 1). Dedicated circuitry is used for controlling the LED duty cycles, selection of stimuli, response monitoring, and for elapsed-time-counting operations. Six integrated circuits are required to implement these functions. All counting and timing operations are handled by a pair of 8253 programmable interval timers. The use of the 8253 timers minimizes computer participation in timing and counting operations and thereby frees the CPU for the performance

The opinions or assertions contained herein are the private views of the authors and are not to be construed as official or as reflecting the views of the Department of the Army or the Department of Defense. Human subjects participated in these studies after giving their free and informed consent. Investigators adhered to AR 70-25 and USAMRDC REG 70-25 on the use of volunteers in research.

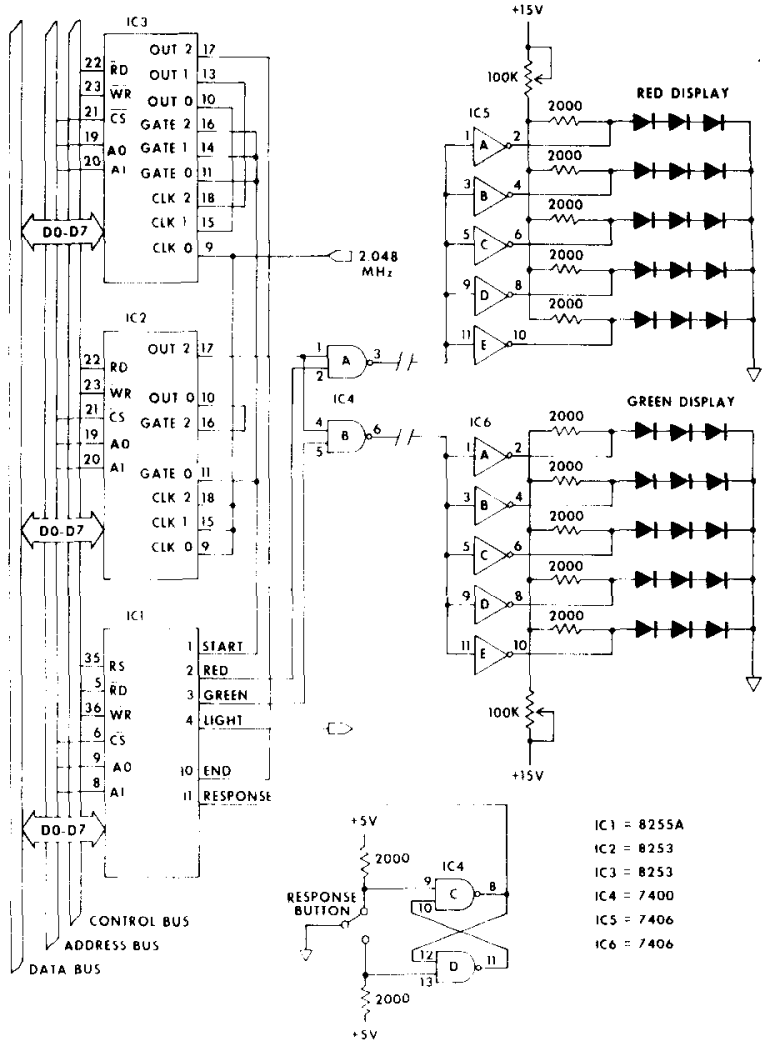

Figure 1. Schematic of the adaptometer control circuitry and stimulus display elements.

of other tasks. Stimulus control and response monitoring operations are performed by an $8255 \mathrm{~A}$ programmable peripheral interface (PPI). The use of the 8253 and $8255 \mathrm{~A}$ programmable integrated circuits greatly simplifies the design and fabrication of the LED adaptometer. With these devices, the control of stimulus parameters and the selection of psychophysical test methods are under software control and are easily changed to meet test requirements. The numerous ports of the PPI also permit the control of complex stimulus displays. A 
7400 NAND gate is used for response button debouncing and as a line driver between the control board and stimulus display. The LED display elements are driven by 7406 hex inverter drivers with open-collector highvoltage outputs.

Each 8253 contains three independent 16-bit downcounters (CNT 0-2) that may be operated at clock frequencies up to $2 \mathrm{MHz}$. Each counter is provided with a clock input (CLK 0-2), an output (OUT 0-2), and a gate (GATE 0-2) input that can be used to reset, stop, or start the counter. The counters can be programmed to operate in several different modes. In the present application, the counters are used as retriggerable one-shots and as divide-by- $\mathrm{N}$ counters. Once programmed, the counters perform their assigned tasks without further supervision by the computer.

The 8255A PPI is a general-purpose 24-line input/ output device. The PPI input/output pins are organized as three 8-bit ports (A, B, and C), the functions of which are under the control of the microprocessor. The LED adaptometer uses Port $A$ of the $8255 \mathrm{~A}$ as a latched output device. In this mode, a control word output to the port activates selected bits which can then be used to control the operation of the other devices on the interface board. The bit pattern will be retained until the next data byte is sent to the PPI. The outputs are used to synchronize timing operations, to control an adaptation light, and for selection of stimulus display elements. Port C of the PPI is programmed for input operation and is used to monitor the status of the response button. Port B is not used in this application.

The 8253 designated IC2 generates the LED duty cycles and flash rates. CNT 0 of IC 2 controls the LED flash rate. The CLK 0 input to this counter is derived from the 2.048-MHz system clock which services the host microcomputer. Operation of CNT 0 is controlled via GATE 0 by the "start" output pin of the PPI. This counter, which is progammed as a rate generator, divides the clock input by 20,480 to produce a $100-\mathrm{Hz}$ output at OUT 0 . The duty cycle of the LEDs is controlled by IC2, CNT 2, which is programmed as a retriggerable one-shot. In this mode of operation, the counting is triggered by the rising edge of a signal applied to the GATE input. When triggered, the initial programmed count value is decremented at a rate determined by the CLK input $(2.048 \mathrm{MHz})$. The output of the oneshot remains low until the terminal (0) count is reached. The retriggerable feature causes the programmed output pulse to be generated each time the gate input signal appears. In this circuit, the $100-\mathrm{Hz}$ output of CNT 0 is connected to the GATE 2 input. Thus, the basic pulse repetition rate is controlled by the input to GATE 2 and the output pulse durations by the initial count value stored in CNT 2. The durations of the output pulses can be changed by reprogramming the initial count values.

The $100-\mathrm{Hz}$ controlled-duration pulse train from $\mathrm{IC} 2$, OUT 2, is connected to the inputs of NAND gates IC4A and IC4B. The other inputs to these gates are connected to the PPI output control lines labeled "red" and "green." Data sent by the computer to Port $A$ of the $8255 \mathrm{~A}$ can selectively activate these control lines. When either of the lines is activated, the corresponding NAND gate is enabled and the LED duty cycle pulse train is transmitted to the stimulus display.

The 8253 labeled IC3 is used as an elapsed time counter. The CLK 0 input is connected to the $2.048-\mathrm{MHz}$ system clock. CNT 0 divides the CLK 0 input to provide a $100-\mathrm{Hz}$ output at OUT 0 . This $.01-\mathrm{sec}$ time base is input to CLK 1 of CNT 1 and further divided to produce a .1-sec time-base output at OUT 1 . That is the basic time unit used for elapsed time measurements in the present system. CNT 2 of IC3 is programmed to produce an interrupt on reaching a terminal count. In this mode, the counter is loaded with some initial count value. The initial count value is decremented once for each clock pulse appearing at the CLK input. When the terminal count is reached, the OUT 2 pin goes high and remains high until the counter is reprogrammed. The OUT 2 output of IC 3 is connected to the PPI input labeled "end." The computer can read data from Port C of the 8255A, check the status of this input, and determine whether or not the end of the programmed time interval has occurred. The counting operations of the three counters are synchronized by connecting the GATE inputs to the PPI "start" output.

The IC3 elapsed time counter is also used for the timing of delays within the calling program. A special program instruction is available which causes the 8253 counter to latch and hold the current count value without disturbing the counting operation. The stored count can then be read by the computer. The technique is used with the LED adaptometer for measuring the duration of test sessions.

There are two additional connections to the $8255 \mathrm{~A}$. The PPI controls an adaptation light via the output labeled "light." When the output is high, a solid state relay switches a tungsten filament light "on." The light is used to light adapt the person under controlled conditions prior to the start of the dark adaptation test. The remaining "response" connection to Port C of the PPI is used to monitor the status of the response button. The button is debounced by NAND Gates IC4C and IC4D, with the output of the former being connected to one of the PPI inputs. When the pushbutton is released, the input is high. This condition can be recognized by the computer by reading a byte of data from Port $C$ and then masking (ANDing) the result to isolate and test the bit corresponding to the "response" input.

Interfacing of the programmable ICs to the typical microprocessor bus is relatively uncomplicated. Each device is assigned a unique range of four addresses. A base address, decoded from the address bus of the computer with external logic, is connected to the $\overline{\mathbf{C S}}$ (chip select) input of the device. The IC is selected when this input is low. Addresses within the IC are selected according to the inputs appearing at Pins AO and A1. 
The highest address within the device (i.e., when $\mathrm{A} 0$ and Al are both high) is used for programming. The lower addresses are used for communicating data between subsections of the IC and the host computer. The direction of flow of information is controlled by the status of the $\bar{R} \bar{D}$ (read) and $\bar{W} \bar{R}$ (write) pins of the IC. Data and instructions are transmitted as 8-bit words (bytes) over the D0-D7 lines. The +5.V supply of the computer can power the programmable and conventional ICs used in the adaptometer control circuit. Details of the bus interconnections and general interface requirements are provided in the manufacturer's literature and in other readily available literature.

Hewlett-Packard red (HP 5082-4670) and green (HP 5082-4970) rectangular LEDs are used as light sources. LEDs of the same color are arranged in groups of three as vertical bars. Each set of three LEDs is driven by the output of a 7406 inverter driver, as illustrated in Figure 1. Five vertical bars of each color are used as test stimuli in this version of the LED adaptometer. Red and green stimuli are located at the center and at the corners of a square target display (Figure 2). The maximum dimensions of the display subtend an angle of $20 \mathrm{deg}$ at the retina. The red LEDs are enclosed in Kodak Wratten 33 filters to improve the color characteristics of the display elements. All LEDs are covered by neutral-density filters with an optical density (OD) of 1.4 to limit the maximum intensity of the display. The target display assembly is located within a .9-m-diam white hemisphere. The hemisphere is equipped with a chin support and headrest and can be illuminated with a diffuse 110-candela $/ \mathrm{m}^{2}$ white light source.

The full (i.e., 100\%) duty cycle equivalent intensity of the LED display is adjusted so that the first plotted values of the red and green adaptation functions fall between the third and fourth ordinates of the graphs (Figure 3). This initial adjustment is made by using the two $100 \mathrm{~K}$ potentiometers illustrated in Figure 1. When this adjustment is performed with the 1.4-OD filters in place, the peak luminance is approximately $\left(11.6 \times 10^{-6} \mathrm{~lm}\right) /\left(\mathrm{cm}^{2} \mathrm{sr}\right)$ for the red display and $\left(12.26 \times 10^{-6} \mathrm{~lm}\right) /\left(\mathrm{cm}^{2} \mathrm{sr}\right)$ for the green display. The average luminance is equal to the peak luminance times

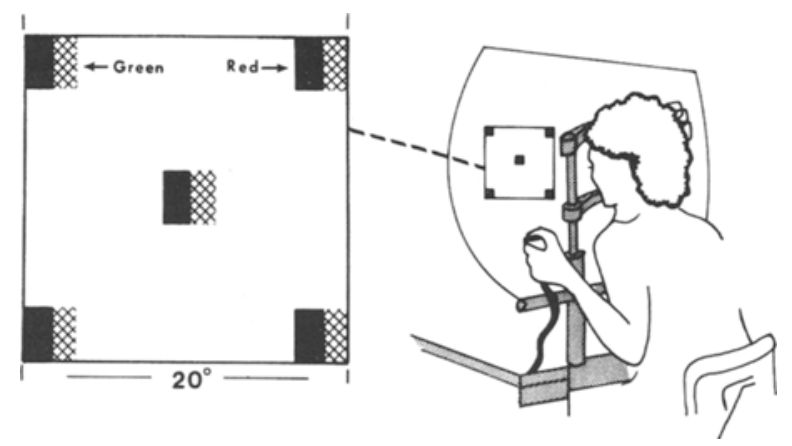

Figure 2. Stimulus display (left) and dark-adaptation apparatus (right).

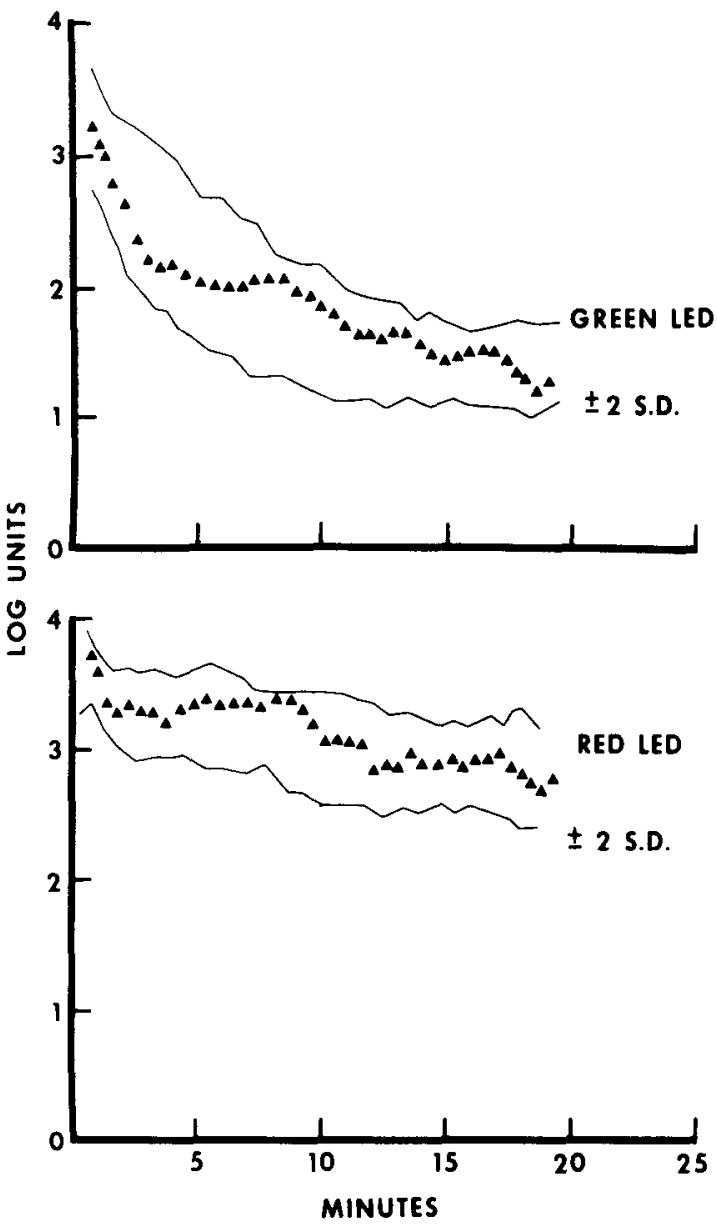

Figure 3. Example of adaptation functions obtained from a normal volunteer using the method of limits and green (upper) and red (lower) stimuli. The adaptation data are bounded by the two standard deviation limits derived from 22 normal volunteers.

the LED duty cycle. Preliminary studies with this instrument have shown that the shapes of the adaptation functions are not changed for starting values within the specified range.

\section{SOFTWARE}

Our "standard" test begins with a 5-min lightadaptation period. The person being tested is then given $20 \mathrm{~min}$ of dark adaptation during which the red and green adaptation functions are derived. The testing begins with a green stimulus presentation. The psychophysical method of limits is used to derive the adaptation functions for the red and green test stimuli. A trial begins by setting the apparent intensity (duty cycle) $.75 \log$ units below the estimated threshold value. The apparent intensity is then incremented in $.05 \mathrm{log}$ steps. Each level is present for $.5 \mathrm{sec}$, during which time the person either fails to detect the stimulus or reports detection by pressing the response button. After the stimulus is detected, the duty cycle is decremented at the rate of $.05 \log$ units $/ .5 \mathrm{sec}$ until the person signals by 
release of the button that it is no longer present. The absolute threshold for each trial is the average of the log values at which response categories changed during the ascending and descending series.

The software used in our laboratory allows the operator to change any of the dark-adaptation test parameters through commands entered at the video terminal. Software options include (1) exponential smoothing of the plotted adaptation functions, (2) detection of response errors, (3) use of a fixation point with automatically adjusted intensity, (4) selection of different psychophysical test methods, and (5) creation of data files on magnetic disk for subsequent statistical analyses.

An abbreviated version of a BASIC program that uses the method of limits to derive separate adaptation functions for the red and green LED test stimuli is listed in the Appendix. This program was written for use with a Heath Company H8 8080A microcomputer using the Heath Disk Operating System (HDOS) version of Microsoft BASIC. Data are plotted on-line using a Houston Instrument HiPlot digital plotter. A reproduction of the graphic output is presented in Figure 3.

This program stores the count values that are used by the 8253 timer (IC2) to control the LED duty cycles. The array $L(N)$ contains 81 count values covering a 4-log-unit range in increments of $.05 \mathrm{log}$ units. The shortest duty cycle is approximately 1 microsec $(2$ counts for the 2.048- $\mathrm{MHz}$ clock input) and the longest is 10,000 microsec $(20,480$ counts). The table entries are computed in lines 1330-1370. The expression in line 1340 derives the log duty cycle values and converts them to integer multiples of the system clock frequency. The log count value is then subtracted from a constant and stored in the array $L(N)$. This operation accomplishes two objections. First, the log count value is corrected for the time required for the LED to emit light after it is activated. LED on-time delays of 2.5 microsec (five clock cycles) were observed in this system. Second, the subtraction of the value $L$ from the constant performs a software inversion of the 8253 output to provide the correct signal polarity to the other integrated circuits in the system. The value of the constant is the number of clock pulses occurring during the interflash intervals minus the five-count correction.

The integer expression in line 1340 limits the precision with which the constant log interval can be approximated. The values computed in line 1350 and stored in the array $P(N)$ are the equivalent $\log$ values (times 100) of the integer counts stored in $L(N)$. The premultiplication of the data stored in $\mathrm{P}(\mathrm{N})$ scales the numbers for use during plotting.

Initialization of the programmable integrated circuits is accomplished by the instructions in lines 1400-1510. Each device is assigned four consecutive addresses, with the highest address receiving the operating mode instructions. The three lower addresses are assigned to the individual counters $(8253 \mathrm{~s})$ or ports $(8255 \mathrm{~A})$ of the device and are used for the exchange of data. The pro- gram begins with the output of an operating mode instruction that defines the task to be performed by one of the counters or ports. The mode control word contains the operating instructions and the coded address of one of the functional units within the device. Each counter or port is programmed separately. The 8253 counters also require initial count values. These are written to the appropriate lower address as 1 or 2 bytes of data.

The 8255A (IC1 addresses 0-3) is instructed to perform simple input/output operations in line 1410. Subsequently, data output to Port A (address 0 ) will be held by the $8255 \mathrm{~A}$ until reprogrammed. Similarly, by reading data from address 2 , the current status of the Port $\mathrm{C}$ input pins can be examined. Data appearing at the input pins of IC1 are not latched when using this mode of operation.

The IC2 (addresses 16-19), CNT 0, is programmed for 16-bit binary divide-by-N operation by the instructions in line 1430 and CNT 2 is programmed for retriggerable one-shot operation, in which the output duration is also controlled by a 16-bit number. A 16-bit quantity cannot be written to a 8253 count register in a single output statement. The low and high bytes of the 16-bit count value must be written during two consecutive output instructions. The decimal equivalents of the low and high bytes of the flash rate divisor $(20,480)$ are output to IC2, CNT 0, in line 1450 . The initial count value for CNT 2 is provided later in the program.

The operating modes of the three IC3 (addresses 8-11) elapsed-time counters are defined by the instructions in line 1470. CNT 0 and CNT 1 are programmed to perform divide-by- $\mathrm{N}$ operations using 16-bit and 8-bit initial count values. The operating mode of CNT 2 causes this counter to count down from some 16-bit initial count and provides an output signal when the terminal count is reached. Initial count values are provided to each counter by the instructions in lines 1490 and 1510. The initial count value output to CNT 3 (address 10) determines the duration of the lightadaptation period. In this program, the initial count of 3,000 produces $5 \mathrm{~min}$ of light adaptation when using the .1-sec time base.

The output control function of the $8255 \mathrm{~A}$ is illustrated by the instruction in line 1560 . Here the number 9 is output to Port A (address 0 ) of IC1. The binary representation of this number is 00001001 . Figure 1 shows that the bits of the binary number in the first and fourth positions (from the right) will activate the "start" and "light" output pins of IC1. This instruction turns on the adaptation light and starts the elapsedtime counters in IC3. In line 1580, data are input continuously from the $8255 \mathrm{~A}$ until the bit corresponding to the output of CNT 2, IC3, goes high upon termination of the elapsed-time count. The output of CNT 2 is connected to the most significant bit of Port $\mathrm{C}$ of the $8255 \mathrm{~A}$. The decimal equivalent of this bit position is 128. The bit is isolated by ANDing the Port $\mathrm{C}$ input 
with 128 . If the input is high, the result will be 128 and the program will exit the loop defined by line 1580 .

The software implementation of the method of limits derivation of the dark-adaptation functions is found in lines 1670-1860. Each stimulus presentation starts with an ascending series. The starting duty cycle value is set $.75 \log$ units below the last threshold (arbitrarily set to $2.5 \mathrm{log}$ units on the first presentation) and the appropriate LED display is switched on. The program then enters a loop, during which the status of the response button is evaluated. The constant D controls the observation interval for each duty cycle presentation. The loop execution time was found to be $.00767 \mathrm{sec}$; When $\mathrm{D}=65$, the observation interval is approximately $.5 \mathrm{sec}$. If no response occurs within $.5 \mathrm{sec}$, the duty cycle is increased to the next highest value and the procedure is repeated. If a response is made, the program stores the current log duty cycle value. The duty cycles are then incrementally decreased until the response button is released. The corresponding $\log$ duty cycle value is again recorded. The absolute threshold for the trial is the average of the log duty cycles recorded during the ascending and descending limbs of the series (line 1860).

After each threshold estimate is obtained, the LED display is switched off (line 1890) and the results are plotted (Figure 3 ) by using the subroutine beginning with line 2150 . The status of the elapsed-time bit of PPI Port $\mathrm{C}$ is checked after each data point is plotted. If the programmed time interval has expired, an endof-session message is written to the video terminal and all the LEDs are switched "on" to alert the person that the test has ended. If the test has not ended, the color of the display is changed and program execution resumes at line 1970.

The elapsed-time counter is accessed by the subroutine beginning with line 2370 . The instruction in line 2380 is used to latch the current count value in the 8253. This operation does not interfere with the count operation. Since the 8253 is programmed as a 16-bit binary counter, it is necessary to read the count register as 2 bytes of data. This is accomplished in line 2390 , where the decimal value is reconstructed from the low and high data bytes and assigned to the variable SEC. Time data are used when plotting the adaptation functions and are stored along with the threshold data on magnetic disks.

\section{SUMMARY}

The design of a low-cost microprocessor-controlled LED display for use in dark-adaptation testing has been presented. This design allows manipulation of stimulus parameters and test protocols through simple changes in software. The results obtained with the LED adaptom. eter are consistent with previously recorded results obtained when using quasi-monochromatic light sources. The present hardware design will support other visual psychophysical experiments involving color, intensity, and spatial information.

\section{REFERENCE NOTE}

1. Zwick, H. J., Biggs, S. L., O'Mara, P. A., \& Van Sice, C. W. A solid-state adaptometer. Proceedings of the 1980 Army Science Conference. West Point, N.Y: June 1980.

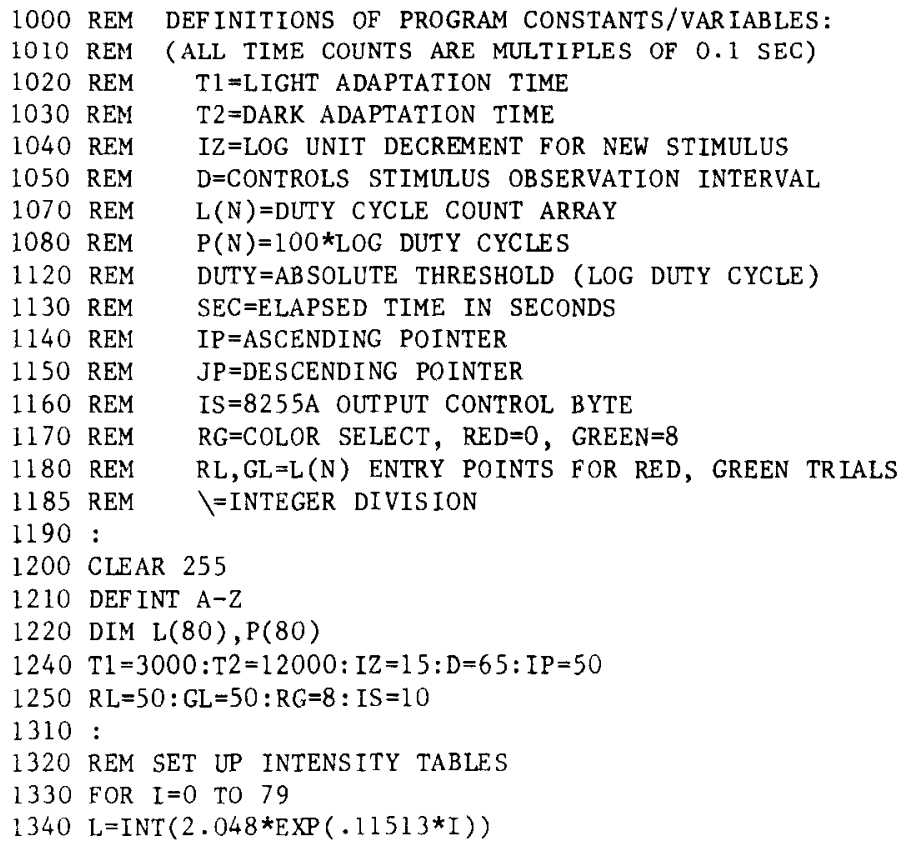


$1350 \mathrm{~L}(\mathrm{I})=20475-\mathrm{L}: \mathrm{P}(\mathrm{I})=\operatorname{INT}(100 * \operatorname{LOG}(\mathrm{L} / 2.048) / 2.30259)$

1360 NEXT I

$1370 \mathrm{~L}(80)=0: P(80)=400$

1380 :

1390 REM HARDWARE INITIALIZATION

1400 REM SET 8255A MODE: PORT A=OUT, PORT C=INPUT

1410 OUT 3,139

1420 REM INITIALIZE DUTY CYCLE TIMERS (8253, IC2)

1430 OUT 19,52 :OUT 19,178

1440 REM SET FLASH RATE TO $100 \mathrm{HZ}$

1450 OUT $16,0:$ OUT 16,80

1460 REM INITIALIZE ELAPSED TIME COUNTERS (8253, IC3)

1470 OUT 11,52:OUT 11,84:OUT 11,176

1480 REM SET 0.1 S TIME BASE $(10 * 10 \mathrm{MS}=0.1 \mathrm{~S})$

1490 OUT 8,0:0UT 8,80:OUT 9,10

1500 REM SET LIGHT ADAPTATION TIME TO Tl

1510 OUT 10,T1 MOD 256:OUT 10,T $\backslash 256$

1520 :

1550 REM BEGIN LIGHT ADAPTATION

1560 OUT 0,9

1570 REM CHECK FOR END OF LIGHT ADAPTATION

1580 IF (INP(2) AND 128) $=128$ GOTO 1590 ELSE GOTO 1580

1590 REM SET DARK ADAPTATION TIME TO T2

1600 OUT 10,T2 MOD 256:OUT 10,T2\256

1650 :

1670 REM SET INITIAL DUTY CYCLE, TURN ON TARGET

1680 OUT $18, \mathrm{~L}$ (IP) MOD 256:OUT 18,L(IP) $256:$ OUT 0 , IS

1690 REM INCREASE DUTY CYCLE EVERY $D * 0.00767 \mathrm{~S}(0.5 \mathrm{~S}$ INTERVAL)

1700 FOR I=1 TO D: IF (INP(2) AND 64) $=0$ THEN 1730 ELSE NEXT I

1710 IP $=$ IP+1 : IF IP $>80$ THEN DUTY $=400:$ GOTO 1880

1720 OUT 18,L(IP) MOD 256:OUT 18,L(IP) \256:GOTO 1700

$1730 \mathrm{JP}=\mathrm{IP}$

1740 REM DECREASE DUTY CYCLE EVERY $D * 0.00767 \mathrm{~S}$ (0.5 S INTERVAL)

1750 FOR I=1 TO D:IF (INP(2) AND 64) $=64$ THEN 1860 ELSE NEXT I

$1760 \mathrm{JP}=\mathrm{JP}-1$ : IF JP $\angle 0$ THEN DUTY $=0$ : GOTO 1880

1770 OUT 18,L(JP) MOD 256:OUT 18,L(JP)\256:GOTO 1750

1850 REM GET THE AVERAGE LOG (GEOMETRIC MEAN OF DUTY CYCIES)

1860 DUTY $=(P($ IP $)+P(J P)) \backslash 2$

1870 :

1880 REM TURN OFF TARGET, GET TIME, PROCESS DATA

1890 OUT $0,8:$ GOSUB 2370

1900 REM STORE THE NEW LOOKUP VALUE

1910 IF RG=0 THEN RL=DUTY \5-IZ: IF RL $<0$ THEN RL=0

1920 IF RG=8 THEN GL=DUTY $\backslash 5-I Z:$ IF $G L<0$ THEN GL=0

1930 REM PLOT DUTY CYCLE VS. TIME

1940 GOSUB 2150

1960 REM CHANGE COLOR AND CONTINUE

1970 IF $R G=0$ THEN $R G=8:$ IS $=10:$ IP $=$ GL: GOTO 1670

1980 IF RG=8 THEN RG=0:IS=12:IP=RL: COTO 1670

1990 :

2010 END

2050 :

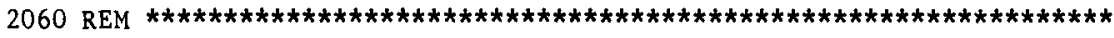

2070 REM SUBROUTINES

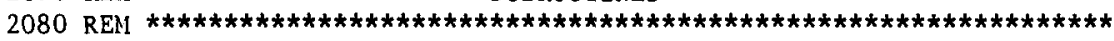

2090 :

2150 REM PLOT THRESHOLD DATA

2160 REM (THE PLOT ROUTINE IS NOT INCLUDED IN THIS LISTING)

2280 RETURN

2290 :

2370 REM GET TIME, CHECK FOR END OF SESSION

2380 OUT 11,128

2385 IF (INP(2) AND 128) $=128$ GOTO 2010

$2390 \mathrm{SEC}=(\mathrm{T} 2-(\operatorname{INP}(10)+256 * \operatorname{INP}(10))) \backslash 10$

2400 RETURN

2440 :

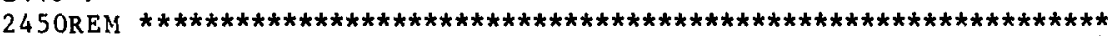

